\title{
Vancomycin-Associated Acute Kidney Injury in a Large Veteran Population
}

\author{
Geeta Gyamlani $^{a}$ Praveen K. Potukuchi ${ }^{b, c}$ Fridtjof Thomas ${ }^{d}$ Oguz Akbilgic ${ }^{e}$ \\ Melissa Soohoo ${ }^{f}$ Elani Streja ${ }^{f}$ Adnan Naseer ${ }^{a}$ Keiichi Sumida ${ }^{b}$ \\ Miklos Z. Molnar b, g, h Kamyar Kalantar-Zadeh ${ }^{f}$ Csaba P. Kovesdy ${ }^{a, b}$ \\ ${ }^{a}$ Nephrology Section, Memphis Veterans Affairs Medical Center, Memphis, TN, USA; ${ }^{b}$ Division of Nephrology, \\ University of Tennessee Health Science Center, Memphis, TN, USA; ${ }^{C}$ IHOP, College of Graduate Health Sciences,

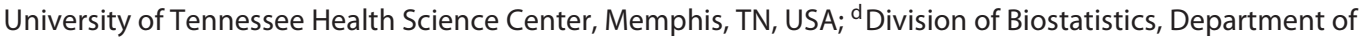

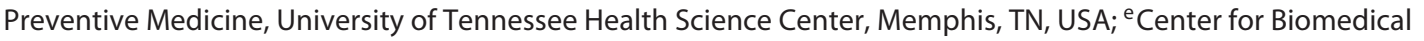 \\ Informatics, Department of Pediatrics, University of Tennessee Health Science Center, Memphis, TN, USA; ${ }^{f}$ Division \\ of Nephrology, University of California, Irvine, CA, USA; ${ }^{9}$ Division of Transplant Surgery, Methodist University \\ Hospital Transplant Institute, Memphis, TN, USA; hivision of Transplant Surgery, Department of Surgery, University \\ of Tennessee Health Science Center, Memphis, TN, USA
}

\section{Keywords}

Acute kidney injury · Vancomycin · Linezolid ·

Daptomycon · Outcomes

\begin{abstract}
Background: To determine the association of vancomycin with acute kidney injury $(\mathrm{AKI})$ in relation to its serum concentration value and to examine the risk of $\mathrm{AKI}$ in patients treated with vancomycin when compared with a matched cohort of patients receiving non-glycopeptide antibiotics (linezolid/daptomycin). Methods: From a cohort of $>3$ million US veterans with baseline estimated glomerular filtration rate $\geq 60 \mathrm{~mL} / \mathrm{min} / 1.73 \mathrm{~m}^{2}$, we identified 33,527 patients who received either intravenous vancomycin $(n=22,057)$ or nonglycopeptide antibiotics (linezolid/daptomycin, $n=11,470$ ). We examined the association of the serum trough vancomycin level recorded within the first $48 \mathrm{~h}$ of administration with subsequent $\mathrm{AKI}$ in all patients treated with vancomycin and
\end{abstract}

\section{KARGER}

(c) 2019 S. Karger AG, Basel

E-Mail karger@karger.com

www.karger.com/ajn association of vancomycin vs. non-glycopeptide antibiotics use with the risk of incident AKI. Results: The overall multivariable adjusted ORs of $A K I$ stages 1, 2, and 3 in patients on vancomycin vs. non-glycopeptides were 1.1 (1.1-1.2), 1.2 (11.4), and 1.4 (1.1-1.7), respectively. When examined in strata divided by vancomycin trough level, the odds of AKI were similar or lower in patients receiving vancomycin compared to non-glycopeptide antibiotics as long as serum vancomycin levels were $\leq 20 \mathrm{mg} / \mathrm{L}$. However, in patients with serum vancomycin levels $>20 \mathrm{mg} / \mathrm{L}$, the ORs of AKI stages 1,2 , and 3 in patients on vancomycin vs. non-glycopeptide antibiotics were 1.5 (1.4-1.7), 1.9 (1.5-2.3), and 2.7 (2-3.5), respectively. Conclusions: Vancomycin use is associated with a higher risk of AKI when serum levels exceed $>20 \mathrm{mg} / \mathrm{L}$.

(c) 2019 S. Karger AG, Basel

G.G. and P.K.P. contributed equally to the manuscript.
Csaba P. Kovesdy, MD Nephrology Section Memphis VA Medical Center 1030 Jefferson Ave., Memphis, TN 38104 (USA) E-Mail ckovesdy@uthsc.edu 


\section{Introduction}

Vancomycin is a commonly used tricyclic glycopeptide antibiotic, which is considered the antibiotic of choice primarily for infections involving methicillin-resistant Staphylococcus aureus (MRSA) [1]. In the past, clinicians were reluctant to prescribe vancomycin due to concerns of product purity, with many clinicians referring to it as "Mississippi mud" [2]. These impurities were associated with adverse effects including acute kidney injury (AKI). Although current preparations purified by high-performance liquid chromatography are $90-95 \%$ pure [3], even today, the generic formulations of vancomycin pose a risk for containing fermentation by-products that could induce AKI $[4,5]$.

In patients who have invasive MRSA infections, in order to be able to achieve a 24 -h ratio of area under the curve to minimum inhibitory concentrations (AUC/ MIC) $>400$ [6], guidelines recommend more intensive dosing and maintenance of trough levels between 15 and $20 \mathrm{mg} / \mathrm{L}$ [7-9]. This recommendation has been widely adopted into clinical practice and may have resulted in an increase in vancomycin-associated AKI [10-12]. There is significant variability in the reported incidence of vancomycin-related AKI, ranging anywhere from $5 \%$ to as high as $43 \%$ depending on the study population and baseline risk factors $[12,13]$. Previous studies examining the association of vancomycin with AKI relied on observations from relatively small cohorts of patients, and thus resulting in uncertainty about the causal role of vancomycin in $\mathrm{AKI}$ and about what strategies to use to prevent AKI in patients treated with vancomycin $[14,15]$.

We conducted a large retrospective study to assess the association between vancomycin administration and the risk of AKI. The purpose of our study was (1) to determine the association of vancomycin with AKI in relation to its early trough serum concentration value and (2) to examine the risk of AKI in patients treated with vancomycin when compared with a matched cohort of patients receiving non-glycopeptide antibiotics.

\section{Materials and Methods}

\section{Study Setting and Cohort Definition}

The institutional review committees at the Memphis and Long Beach Veterans Affairs Medical Centers approved the study. Data were obtained from the Racial and Cardiovascular Risk Anomalies in chronic kidney disease (CKD; RCAV) study, which examines risk factors of incident CKD, mortality, and other outcomes in US veterans, and which was previously described in detail $[16,17]$.
From an original cohort of 3,582,478 patients with baseline estimated glomerular filtration rate $(\mathrm{eGFR}) \geq 60 \mathrm{~mL} / \mathrm{min} / 1.73 \mathrm{~m}^{2}$, we included patients who received vancomycin for at least $48 \mathrm{~h}$ during an inpatient hospitalization (considering only the first hospitalization for patients with multiple such events), who had sufficient serum creatinine measurements for AKI diagnosis and who had no AKI events and at least one steady state trough serum vancomycin concentration available in first $48 \mathrm{~h}$. From the same cohort, we also identified patients who received non-glycopeptide antibiotics (intravenous daptomycin and/or linezolid) without IV vancomycin and who had no AKI events in the first $48 \mathrm{~h}$ (Fig. 1).

\section{Exposures and Covariates}

All data (including patient characteristics, laboratory variables, and medication exposures) were extracted from various national VA research databases, as previously described [16-19]. Baseline characteristics were identified on the first day of antibiotic administration or up to 7 days before it (using the value closest to the date of antibiotic administration). We identified antibiotic (vancomycin and non-glycopeptides) exposure from VA pharmacy dispensation records, which also included the number of doses and the duration of each dose. The main exposure variable was the highest recorded trough vancomycin level measured within the first $48 \mathrm{~h}$ after initiation of therapy, with the mean of trough levels measured within the first $48 \mathrm{~h}$ after initiation of therapy used in sensitivity analyses. We assessed vancomycin levels measured during the first $48 \mathrm{~h}$ in patients without AKI events during this time period in order to avoid reverse causation, since AKI can result in a subsequent rise in vancomycin levels. Baseline eGFR was calculated from serum creatinine measurements using the CKD Epidemiology Collaboration Equation [20]. Information about comorbidities was collected from the VA Inpatient and Outpatient Medical SAS Datasets using ICD-9-CM diagnostic and procedure codes and Current Procedural Terminology codes.

Outcomes

The primary outcome of interest was incident AKI. AKI was defined as an absolute increase in serum creatinine of $\geq 0.3 \mathrm{mg} / \mathrm{dL}$ or $\mathrm{a} \geq 50 \%$ increase in serum creatinine from baseline. AKI was also staged according to KDIGO creatinine-based criteria from the date of first antibiotic exposure during hospitalization [21]. Baseline creatinine was defined as the mean of outpatient serum creatinine levels 7-365 days prior to hospitalization [22]. For our analyses, we only considered AKI events occurring after $48 \mathrm{~h}$ following the start of vancomycin administration.

\section{Statistical Analysis}

Data were summarized using proportions, means $\pm \mathrm{SD}$, or medians (interquartile range) as appropriate. We examined the association of the highest trough serum vancomycin level recorded in the first $48 \mathrm{~h}$ of administration with subsequent $\mathrm{AKI}$ in all patients treated with vancomycin using multivariable adjusted logistic regression. Patients who received vancomycin were categorized into 4 groups based on their trough vancomycin level $(<10,10-15$, $15.1-20$, and $>20 \mathrm{mg} / \mathrm{L}$ ). Variables entered in multivariable-adjusted models were selected based on theoretical considerations; we included variables known to be associated both with vancomycin use and AKI based on scientific evidence, and which were available in our database. Models were adjusted sequentially for the following confounders: model 1: unadjusted; model 2: demo- 


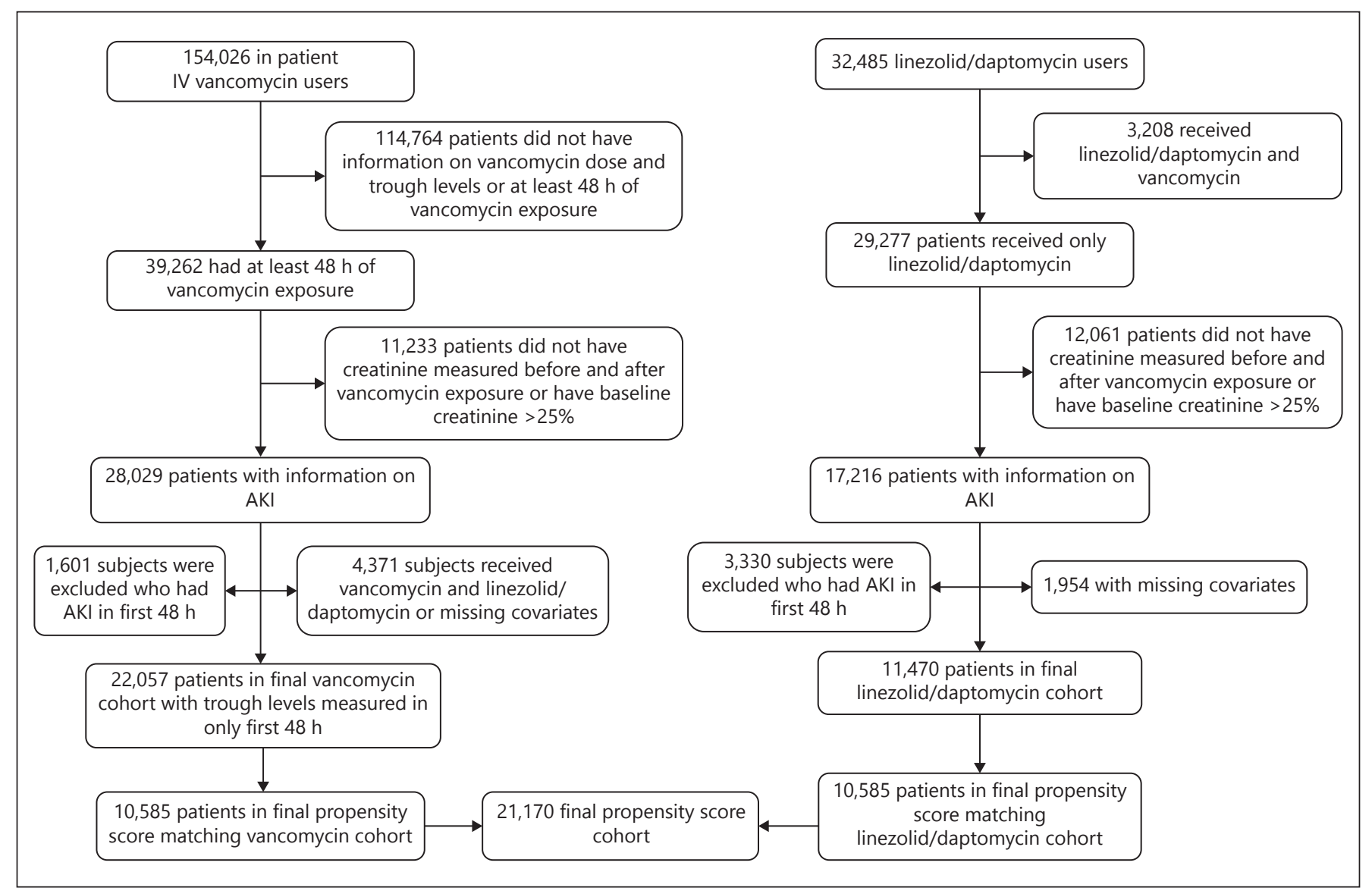

Fig. 1. Flow chart of patient selection. AKI, acute kidney injury.

graphic characteristics; model 3: model 2 variables plus comorbidities; and model 4: model 3 variables plus medications, systolic/ diastolic blood pressure, and body mass index. We performed additional adjustments for select Sequential Organ Failure Assessment score components (platelet count, bilirubin, and vasopressor/ionotropic medications, with and without the addition of arterial pO2; model $5-$ pO2 and + pO2) when these were available. Due to the low proportion of patients in whom the Sequential Organ Failure Assessment variables were available (76\% missing for model $5+$ pO2 and $35 \%$ missing for model $5-$ pO2), these adjustments were considered sensitivity analyses. We also performed subgroup analyses in select subgroups of patients receiving different doses ( $<4$ vs. $\geq 4 \mathrm{~g} /$ day), duration ( $<7$ vs. $\geq 7$ days) of vancomycin therapy, and number of antibiotics using our model 4.

To attenuate bias by indication, we matched patients who received vancomycin with patients who were treated with non-glycopeptides, which are typically employed for indications that are similar to the ones used for vancomycin therapy. We matched patients in the 2 groups (vancomycin vs. non-glycopeptide) by propensity scores calculated from patient demographics, comorbidities, baseline eGFR, mean arterial pressure, and nephrotoxic medication exposure (analgesics, antibiotics, inotropes/pressors, proton pump inhibitors, and diuretics). We used a 1:1 nearestneighbor matching without replacement first in all antibiotic-

Vancomycin and AKI treated patients, and then we repeated the matching process for the 4 subgroups of patients with different vancomycin levels $(<10$ through $>20 \mathrm{mg} / \mathrm{L}$ as described above). Differences between variables were examined by calculating standardized differences, with values $<0.1$ deemed acceptable.

We repeated analyses after excluding patients who died during the time period used to evaluate the occurrence of AKI.

Statistical analyses were performed using SAS Enterprise Guide 7.1 (SAS Institute Inc., Cary, NC, USA) and Stata version 15 (Stata Corporation, College Station, TX, USA).

\section{Results}

\section{Baseline Characteristics}

Overall baseline characteristics in patients categorized by their highest trough vancomycin levels and in propensity-matched patients are shown in Tables 1 and 2. The mean (SD) age of the cohort was 68 (11) years, mean eGFR was 76 (26) $\mathrm{mL} / \mathrm{min} / 1.73 \mathrm{~m}^{2}$, 96\% were male, $19 \%$ were African-American, 50\% were diabetic, and $85 \%$ 
Table 1. Baseline characteristics all the patients who received vancomycin divided into various groups categorized by maximum trough vancomycin levels

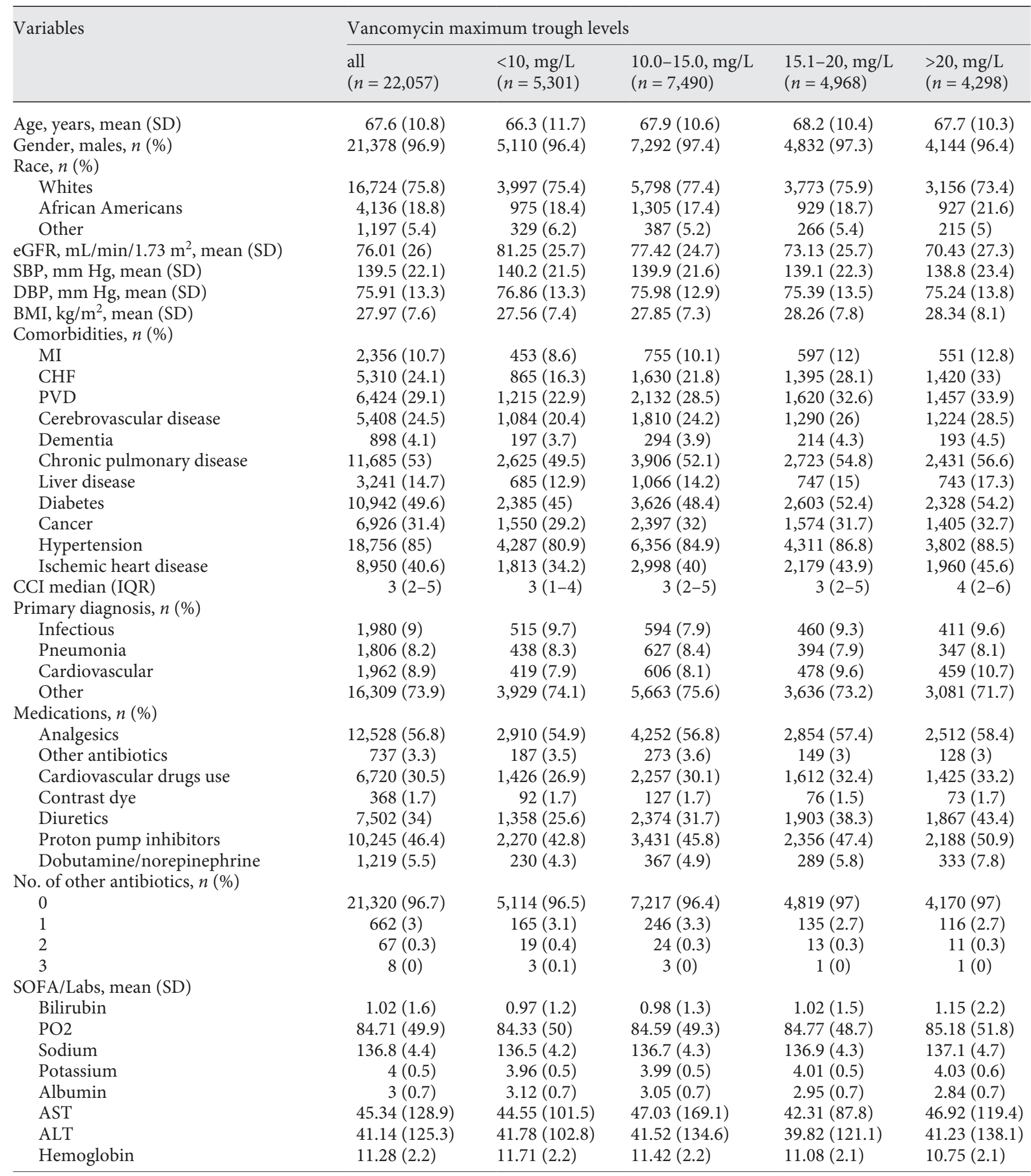


Table 1. (continued)

\begin{tabular}{|c|c|c|c|c|c|}
\hline \multirow[t]{2}{*}{ Variables } & \multicolumn{5}{|c|}{ Vancomycin maximum trough levels } \\
\hline & $\begin{array}{l}\text { all } \\
(n=22,057)\end{array}$ & $\begin{array}{l}<10, \mathrm{mg} / \mathrm{L} \\
(n=5,301)\end{array}$ & $\begin{array}{l}10.0-15.0, \mathrm{mg} / \mathrm{L} \\
(n=7,490)\end{array}$ & $\begin{array}{l}15.1-20, \mathrm{mg} / \mathrm{L} \\
(n=4,968)\end{array}$ & $\begin{array}{l}>20, \mathrm{mg} / \mathrm{L} \\
(n=4,298)\end{array}$ \\
\hline WBC & $13.53(289.3)$ & $11.75(7)$ & $12(59.9)$ & $19.79(605.3)$ & $11.2(7.9)$ \\
\hline Bicarbonate & $25.89(4.3)$ & $25.78(4)$ & $25.92(4.2)$ & $25.96(4.4)$ & $25.91(4.7)$ \\
\hline Platelets & $230.7(119.1)$ & $228.1(114.3)$ & $230.3(116.8)$ & $234.9(127.3)$ & $229.5(118.8)$ \\
\hline
\end{tabular}

Data were summarized using proportions, means $\pm \mathrm{SD}$, or medians (IQR) as appropriate.

AKI, acute kidney injury; CHF, congestive heart failure; PVD, peripheral vascular disease; CVA, cerebrovascular accident; MI, myocardial infarction; eGFR, estimated glomerular filtration rate; BMI, body mass index; CCI, Charlson comorbidity index; SBP, systolic blood pressure; DBP, diastolic blood pressure; SOFA, sequential organ failure assessment; ALT, alanine aminotransferase; AST, aspartate aminotransferase; IQR, interquartile range.

were hypertensives. Comorbidities such as congestive heart failure, peripheral vascular disease, and ischemic heart disease were present in 24,29 , and $41 \%$ of the patients, respectively (Table 1).

\section{Risk of AKI}

Overall crude incidences of AKI stages 1,2, and 3 were $10.4,2.7$, and $1.5 \%$, respectively, among the 22,057 patients who received vancomycin therapy. Figure 2 and online supplementary Table 1 (for all online suppl. material, see www.karger.com/doi/10.1159/000496484) show the association between the highest recorded trough vancomycin levels and incident AKI in unadjusted and adjusted logistic regression models, indicating a linearly higher risk of AKI in patients with higher trough levels. Compared to patients with vancomycin levels $<10 \mathrm{mg} / \mathrm{L}$, patients with levels $>20 \mathrm{mg} / \mathrm{L}$ had a nearly 4 -fold higher multivariable adjusted odds (OR in model 4: 4.1 [3.64.7]) of AKI. The ORs of AKI stages 1,2, and 3 in patients receiving vancomycin vs. non-glycopeptides were 1.1 $(1.1-1.2), 1.2(1-1.4)$, and $1.4(1.1-1.7)$, respectively (Fig. 3 and online suppl. Table 2). In propensity-matched subgroups of patients with plasma vancomycin levels $\leq 20$ $\mathrm{mg} / \mathrm{L}$, the odds of various AKI stages were similar or lower in patients who received vancomycin vs. non-glycopeptides (Fig. 3 and online suppl. Table 2). However, in patients with vancomycin levels $>20 \mathrm{mg} / \mathrm{L}$, the risk of all AKI stages was higher in patients who received vancomycin: the ORs $(95 \% \mathrm{CI})$ of AKI stages 1, 2, and 3 in patients exposed to vancomycin vs. non-glycopeptides were 1.5 (1.4-1.7), 1.9 (1.5-2.3), and 2.7 (2-3.5), respectively (Fig. 3 and online suppl. Table 2).

Figure 4 shows the association of vancomycin exposure categorized by highest trough levels with the risk of incident $\mathrm{AKI}$ in patients receiving different doses ( $<4$ vs. $\geq 4 \mathrm{~g} /$ day) and duration ( $<7$ vs. $\geq 7$ days) of vancomycin therapy, and in patients receiving different numbers of concomitantly administered other antibiotics. There was no significant difference in the risk of AKI in the various subgroups.

Online suppl. Figure S-1B shows the association between highest trough vancomycin categories and odds of AKI in additional clinically relevant subgroups of patients, showing qualitatively similar trends except in patients with infections, pneumonia, and cardiovascular and other diseases ( $p<0.05$ for interaction). Results were similar when using the mean of all trough levels to define vancomycin exposure (data not shown).

About 1.2, 1.4, 2.1, and 2.6\% of patients died in the subgroups with serum vancomycin levels of $<10,10-15$, $15.1-20$, and $>20 \mathrm{mg} / \mathrm{L}$, respectively. Results remain unchanged in sensitivity analyses after excluding patients who died (online suppl. Tables 3, 4 and Fig. S1-D, E).

\section{Discussion}

Our study describes an almost fourfold increased risk of AKI in patients with vancomycin trough levels $>20$ $\mathrm{mg} / \mathrm{L}$. The risk of AKI was not increased in patients receiving vancomycin compared to non-glycopeptides (linezolid/daptomycin) as long as plasma vancomycin levels were $\leq 20 \mathrm{mg} / \mathrm{L}$. However, in patients with trough serum vancomycin levels $>20 \mathrm{mg} / \mathrm{L}$, the risk of all stages of AKI was increased. Clinicians are increasingly concerned about MRSA as an infectious threat in the hospital and now in the community [23]. For many decades, vancomycin has been the drug of choice for treatment of MRSA 
Table 2. Characteristics of the propensity-matched cohort

\begin{tabular}{|c|c|c|c|}
\hline & $\begin{array}{l}\text { Non-glycopeptide } \\
(n=10,585)\end{array}$ & $\begin{array}{l}\text { Vancomycin } \\
(n=10,585)\end{array}$ & $\begin{array}{l}\text { Standardized } \\
\text { difference }\end{array}$ \\
\hline Age, years, mean (SD) & $66.1(10.7)$ & $66.1(10.7)$ & 0.0054 \\
\hline Gender, males, $n(\%)$ & $10,239(96.7)$ & 10,233 (96.7) & -0.0032 \\
\hline \multicolumn{4}{|l|}{ Race, $n(\%)$} \\
\hline Whites & 7,927 (74.9) & $7,918(74.8)$ & \multirow[t]{3}{*}{0} \\
\hline African Americans & $2,198(20.8)$ & $2,208(20.9)$ & \\
\hline Other & $460(4.4)$ & $459(4.3)$ & \\
\hline eGFR, $\mathrm{mL} / \mathrm{min} / 1.73 \mathrm{~m}^{2}$, mean $(\mathrm{SD})$ & $70.94(32.2)$ & $71.17(28)$ & 0.0079 \\
\hline $\mathrm{SBP}, \mathrm{mm} \mathrm{Hg}$, mean (SD) & $137.3(22.2)$ & $137.3(22.3)$ & -0.0003 \\
\hline DBP, mm Hg, mean (SD) & $74.65(13.5)$ & $74.65(13.4)$ & 0.0049 \\
\hline $\mathrm{BMI}, \mathrm{kg} / \mathrm{m}^{2}$, mean $(\mathrm{SD})$ & $27.6(7.8)$ & $28.37(7.9)$ & 0.0017 \\
\hline \multicolumn{4}{|l|}{ Comorbidities, $n(\%)$} \\
\hline MI & $1,154(10.9)$ & $1,159(10.9)$ & 0.0015 \\
\hline $\mathrm{CHF}$ & $2,822(26.7)$ & $2,861(27)$ & 0.0083 \\
\hline PVD & $3,406(32.2)$ & $3,379(31.9)$ & -0.0055 \\
\hline CVA & $2,521(23.8)$ & $2,573(24.3)$ & 0.0115 \\
\hline Dementia & $388(3.7)$ & $384(3.6)$ & -0.002 \\
\hline Chronic pulmonary disease & $5,280(49.9)$ & $5,278(49.9)$ & -0.0004 \\
\hline Liver disease & $1,681(15.9)$ & $1,671(15.8)$ & -0.0026 \\
\hline Diabetes & $5,473(51.7)$ & $5,502(52)$ & 0.0055 \\
\hline Cancer & $3,235(30.6)$ & $3,214(30.4)$ & -0.0043 \\
\hline Hypertension & $8,779(82.9)$ & $8,789(83)$ & 0.0025 \\
\hline Ischemic heart disease & $4,471(42.2)$ & $4,525(42.7)$ & 0.0103 \\
\hline CCI median (IQR) & $3(2-5)$ & $3(2-5)$ & -0.0006 \\
\hline \multicolumn{4}{|l|}{ Medications, $n(\%)$} \\
\hline Analgesics & $5,694(53.8)$ & $5,739(54.2)$ & 0.0085 \\
\hline Other antibiotics & $556(5.3)$ & $583(5.5)$ & 0.0113 \\
\hline Cardiovascular drugs use & $3,137(29.6)$ & $3,186(30.1)$ & 0.0101 \\
\hline Contrast dye & $239(2.3)$ & $246(2.3)$ & 0.0044 \\
\hline Diuretics & $4,282(40.5)$ & $4,341(41)$ & 0.0113 \\
\hline Proton pump inhibitors & $5,603(52.9)$ & $5,622(53.1)$ & 0.0036 \\
\hline Dobutamine/norepinephrine & $861(8.1)$ & $864(8.2)$ & 0.001 \\
\hline \multicolumn{4}{|l|}{ No. of other antibiotics, $n(\%)$} \\
\hline 0 & $10,029(94.7)$ & $10,002(94.5)$ & 0.0113 \\
\hline 1 & $491(4.6)$ & $515(4.9)$ & \\
\hline 2 & $59(0.6)$ & $60(0.6)$ & \\
\hline 3 & $6(0.1)$ & $8(0.1)$ & \\
\hline
\end{tabular}

Data were summarized using proportions, means $\pm \mathrm{SD}$, or medians $(\mathrm{IQR})$ as appropriate.

AKI, acute kidney injury; CHF, congestive heart failure; PVD, peripheral vascular disease; CVA, cerebrovascular accident; MI, myocardial infarction; eGFR, estimated glomerular filtration rate; BMI, body mass index; CCI, charlson comorbidity index; SBP, systolic blood pressure; DBP, diastolic blood pressure; SOFA, sequential organ failure assessment; ALT, alanine aminotransferase; AST, aspartate; IQR, interquartile range.

[24]. While vancomycin has been readily utilized and studied for over 6 decades, the dosing safety margins remain ill defined.

Consequently, recent guidelines recommend to maintain vancomycin trough levels of $15-20 \mathrm{mg} / \mathrm{L}$ in certain high-risk infections [25]. Prior smaller studies have suggested that maintaining levels $>15 \mathrm{mg} / \mathrm{L}$ sub- stantially increases the risk of nephrotoxic events $[8$, 26-28]. Results of a meta-analysis suggested that a trough-toxicity gradient exists, with the greatest risk observed among individuals with trough levels $>20$ $\mathrm{mg} / \mathrm{L} \mathrm{[29].} \mathrm{Our} \mathrm{results} \mathrm{corroborate} \mathrm{these} \mathrm{findings} \mathrm{in} \mathrm{a}$ much larger cohort; while we detected a linear association between higher vancomycin levels and increased 


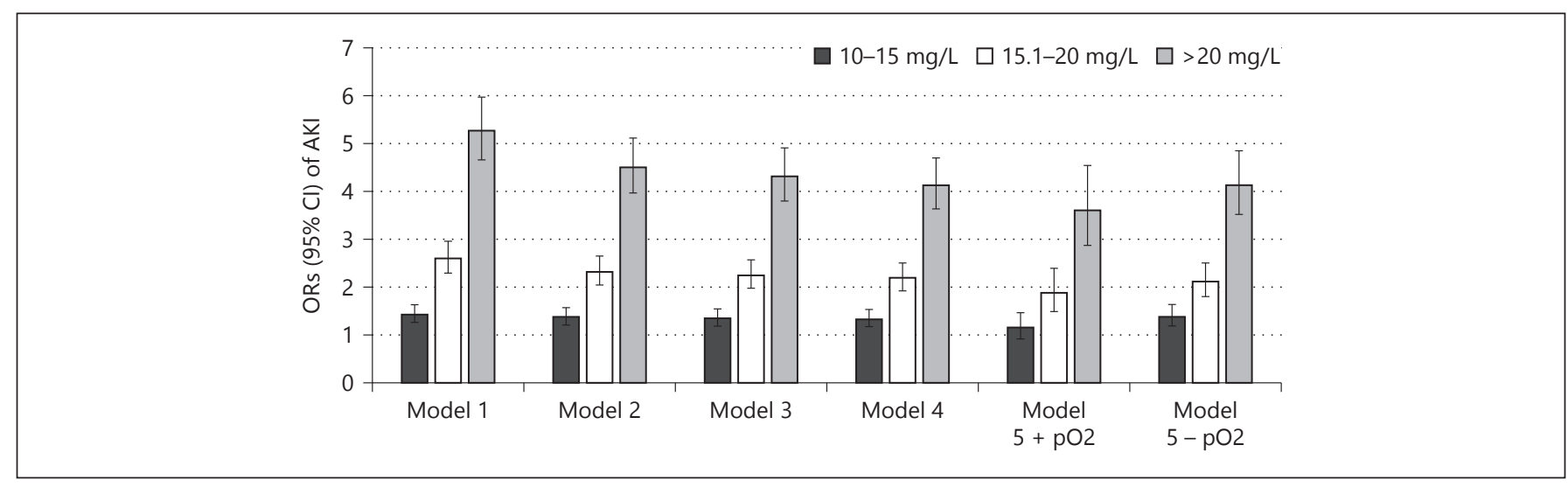

Fig. 2. Association of maximum trough serum vancomycin levels with risk of incident $\mathrm{AKI}$ in adjusted and unadjusted logistic regression models in 22,057 US veteran patients. Model 1: unadjusted; model 2: demographic characteristics; model 3: model 2 variables plus comorbidities; model 4: model 3 variables plus medications, systolic/diastolic blood pressure, and BMI; model 5: model 4 variables and SOFA score components (platelet count, bilirubin, and vasopressor/ionotropic medications, with and without the addition of arterial pO2; model $5-\mathrm{pO} 2$ and + pO2). AKI, acute kidney injury. The group with vancomycin levels $<10 \mathrm{mg} / \mathrm{L}$ served as referent.
Fig. 3. ORs of various stages of AKI (vs. no $\mathrm{AKI}$ ) associated with vancomycin vs. nonglycopeptide use, in propensity scorematched patients overall and in groups categorized by maximum trough serum vancomycin level. AKI, acute kidney injury.

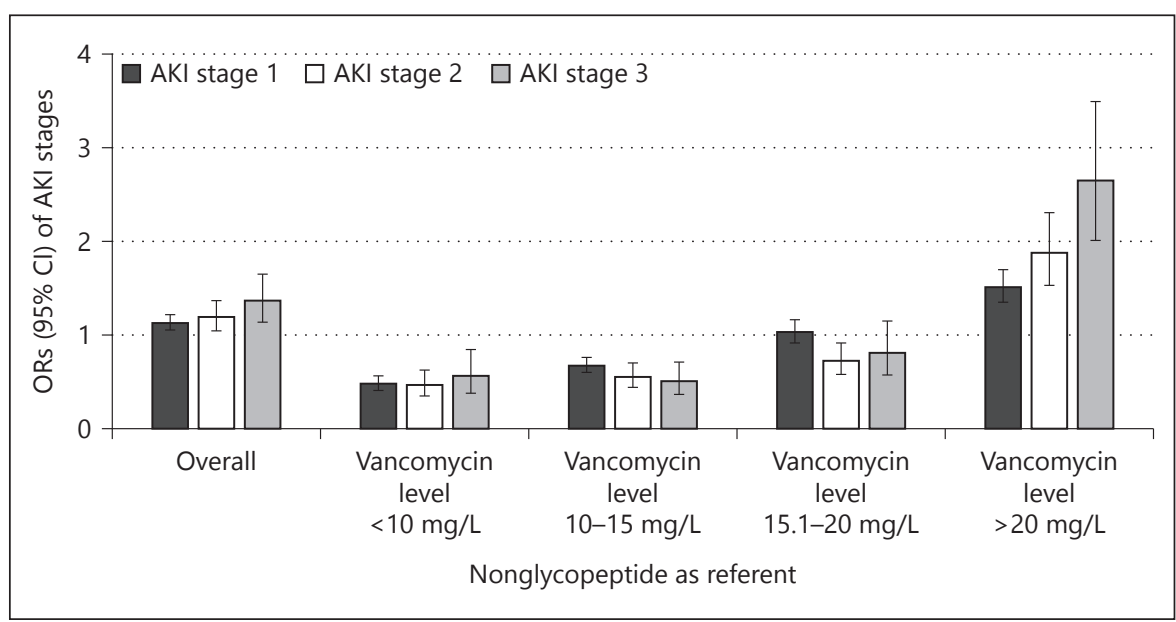

risk of AKI, our propensity-matched analyses comparing vancomycin to non-glycopeptide antibiotic exposure suggest that the risk of AKI may only be present when vancomycin serum levels exceed $20 \mathrm{mg} / \mathrm{L}$. In a comparison trial evaluating the incidence of nephrotoxicity using vancomycin or linezolid for treatment of nosocomial MRSA pneumonia, there was a $9.6 \%$ greater incidence of nephrotoxicity with vancomycin [28].

We did not detect a difference in the risk of AKI in patients exposed to different doses and duration of vancomycin, possibly because we accounted for serum vancomycin levels, which would quantify the deleterious effect of higher doses/longer exposure. In prior studies, vancomycin doses of $\geq 4 \mathrm{~g} /$ day were associated with a higher risk of vancomycin-related nephrotoxicity [30], but the mean vancomycin trough levels were significantly different in patients who received $\geq 4 \mathrm{~g} /$ day. Regarding the duration of exposure in various studies, nephrotoxicity occurred between 4.3 and 17 days after initiation of vancomycin therapy $[10,31,32]$, with vancomycin therapy $\geq 7$ days described as a risk factor for the development of nephrotoxicity in one study [33].

With the growing prevalence of MRSA infections, clinicians need to be alert and recognize the association and the possibility of vancomycin-associated nephrotoxicity [34]. However, it is unclear what component of nephrotoxicity is due to MRSA infection versus vancomycin therapy itself. MRSA is associated with glomerulonephritis and IgA nephropathy. Serious infections 


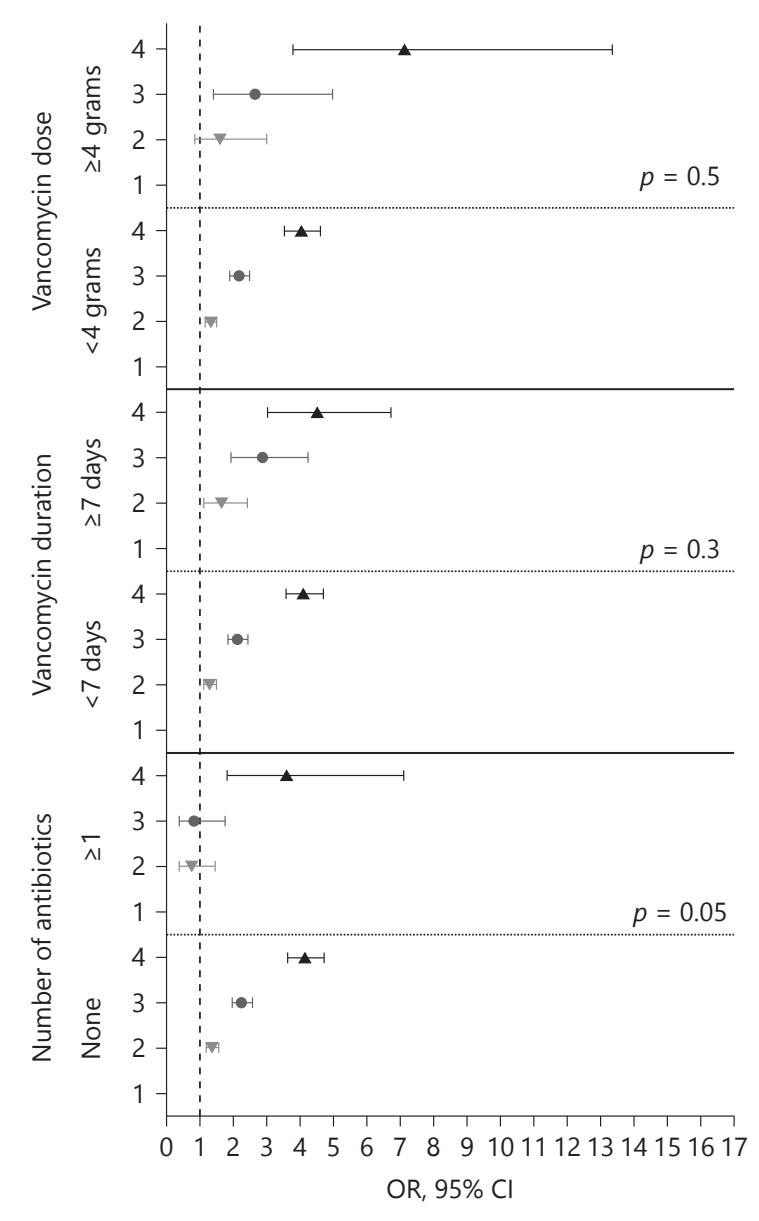

Fig. 4. Association of maximum trough serum vancomycin levels with risk of incident AKI in multivariable adjusted logistic regression models in patients with different doses ( $<4$ vs. $\geq 4$ g/day), duration ( $<7$ vs. $\geq 7$ days), and number of antibiotics. Models were adjusted for patient demographics, BMI, comorbidities, baseline eGFR, mean arterial pressure, and nephrotoxic medication exposure. Patients divided into 4 groups based on the highest recorded serum vancomycin trough level. $1:<10,2: 10-15,3: 15.1-20$, and 4: $>20 \mathrm{mg} / \mathrm{L}$. The group with vancomycin levels $<10 \mathrm{mg} / \mathrm{L}$ served as referent.

such as endocarditis, meningitis, and osteomyelitis often warrant higher therapeutic troughs, thus increasing the risk for vancomycin-associated nephrotoxicity [35, 36]. A previous study reported that lower respiratory tract infections were independently associated with nephrotoxicity, and these effects were independent of initial vancomycin trough levels [37]. Also in our subgroup analysis, there was an association between lower respiratory tract infection and vancomycin-associated AKI.
A recent systematic review and meta-analysis found that the risk for renal injury is compounded when there is co-administration of multiple potentially nephrotoxic agents. This perfect storm could happen when loop diuretics, aminoglycosides, and/or vasopressors are administered concurrently with vancomycin. The analysis demonstrated a high degree of associative risk with a grouped OR (95\% CI) for nephrotoxicity as a whole 3.30 (1.308.39) [29]. Our study was unable to quantify the role of concomitant nephrotoxins in the observed results, primarily because the duration and timing of concomitant nephrotoxins were not quantifiable.

The mechanism whereby vancomycin may cause nephrotoxicity is unclear. Animal data suggest that vancomycin is an oxidative stressor in proximal renal tubular cells [38]. In addition, the use of antioxidants and cilastatin has been found to protect against vancomycininduced kidney damage, further supporting the notion that vancomycin is an oxidative stressor in the proximal renal tubular cells $[39,40]$. Human data also suggest that vancomycin toxicity involves both the proximal tubules and the medullary region of the kidney [41]. However, cases of AKI in humans where a kidney biopsy was performed have mostly shown acute interstitial nephritis $[42-44]$ with acute tubular injury being reported rarely $[45,46]$.

Our study is notable for its large sample size and for being representative of veterans from the entire USA. To the best of our knowledge, this is the largest study to find significant associations between a high vancomycin level and AKI, and one that also includes a control group of patients receiving non-nephrotoxic antibiotics employed for similar indications as vancomycin. Several study limitations also merit discussion. This being an observational study, we can only report associations but not causality. While we adjusted our models for numerous patient characteristics including those quantifying severity of illness, and while we used propensity-matched analyses, we still cannot rule out residual confounding. Our study population consisted of mostly male US veterans; hence, the results should be applied with caution to females and to patients in other countries. Additionally, we excluded patients with CKD, which is a risk factor for vancomycinassociated AKI, which reduces external validity. While the association of higher vancomycin levels with AKI is suggestive of a dose-response effect [27, 31, 47], vancomycin is eliminated predominantly by glomerular filtration, and hence a decrease in renal function in AKI will increase vancomycin plasma concentrations [10] and could result in a spurious association between higher 
trough levels and AKI. By limiting the assessment of vancomycin exposure to measurements performed during the initial $48 \mathrm{~h}$ and by excluding AKI events detected during this time, we minimized the possibility of such reverse causation, although we cannot rule out the effects of undetected AKI events, since rises in serum creatinine follow AKI events with a slight delay. Deviation from vancomycin measurement protocol (e.g., not obtaining levels at $30 \mathrm{~min}$ before the next dose) in individual hospitals may have led to misclassification of the vancomycin stratum.

In conclusion, serum vancomycin levels $>20 \mathrm{mg} / \mathrm{L}$ are associated with significantly higher risk of all stages of AKI. These results have important clinical implications and suggest that serum trough concentrations can serve as a prognostic indicator for nephrotoxicity, and that AKI may be preventable by careful monitoring of vancomycin levels. For conditions that warrant higher vancomycin targets, non-glycopeptide antibiotics may be a viable option in high-risk patients. Clinicians are urged to carefully dose and proactively monitor vancomycin therapy particularly in the most vulnerable patients at greatest risk for developing AKI. Future research direc- tions should focus on early identification of patients at risk for nephrotoxicity and prospective strategies to prevent AKI.

\section{Acknowledgments}

This study is supported by grant R01DK096920 from the National Institute of Health to C.P.K. and K.K.-Z. and by resources from the US Department of Veterans Affairs. Support for VA/ CMS data is provided by the Department of Veterans Affairs, Veterans Health Administration, Office of Research and Development, Health Services Research and Development, VA Information Resource Center (Project Numbers SDR 02-237 and 98004). G.G., A.N., C.P.K., and K.K.-Z. are employees of the Department of Veterans Affairs. Opinions expressed in this paper are those of the authors and do not necessarily represent the opinion of the Department of Veterans Affairs. The results of this paper have not been published previously in whole or in part.

\section{Disclosure Statement}

None of the authors have relevant conflicts of interest.

\section{References}

1 Liu C, et al: Clinical practice guidelines by the infectious diseases society of america for the treatment of methicillin-resistant Staphylococcus aureus infections in adults and children: executive summary. Clin Infect Dis 2011;52:285-292.

2 Levine DP: Vancomycin: a history. Clin Infect Dis 2006;42(suppl 1):S5-S12.

3 Nambiar S, et al: Product quality of parenteral vancomycin products in the United states. Antimicrob Agents Chemother 2012;56: 2819-2823.

4 Tattevin P, et al: Efficacy and quality of antibacterial generic products approved for human use: a systematic review. Clin Infect Dis 2014;58:458-469.

5 Tattevin P, et al: Comparison of six generic vancomycin products for treatment of methicillin-resistant Staphylococcus aureus experimental endocarditis in rabbits. Antimicrob Agents Chemother 2013;57:1157-1162.

6 van Hal SJ, Lodise TP, Paterson DL: The clinical significance of vancomycin minimum inhibitory concentration in Staphylococcus aureus infections: a systematic review and metaanalysis. Clin Infect Dis 2012;54:755-771.

7 Moise-Broder PA, et al: Pharmacodynamics of vancomycin and other antimicrobials in patients with Staphylococcus aureus lower respiratory tract infections. Clin Pharmacokinet 2004;43:925-942.
8 Kullar R, et al: Impact of vancomycin exposure on outcomes in patients with methicillinresistant Staphylococcus aureus bacteremia: support for consensus guidelines suggested targets. Clin Infect Dis 2011;52:975-981.

9 Rybak MJ, et al: Therapeutic monitoring of vancomycin in adults summary of consensus recommendations from the American society of health-system pharmacists, the infectious diseases society of America, and the society of infectious diseases pharmacists. Pharmacotherapy 2009;29:1275-1279.

10 Hidayat LK, et al: High-dose vancomycin therapy for methicillin-resistant Staphylococcus aureus infections: efficacy and toxicity. Arch Intern Med 2006;166:2138-2144.

11 Ingram PR, et al: Risk factors for nephrotoxicity associated with continuous vancomycin infusion in outpatient parenteral antibiotic therapy. J Antimicrob Chemother 2008;62: $168-171$.

12 Jeffres $\mathrm{MN}$, et al: A retrospective analysis of possible renal toxicity associated with vancomycin in patients with health care-associated methicillin-resistant Staphylococcus aureus pneumonia. Clin Ther 2007;29: 1107-1115.

13 Kullar R, et al: Effects of targeting higher vancomycin trough levels on clinical outcomes and costs in a matched patient cohort. Pharmacotherapy 2012;32:195-201.
14 Carreno JJ, et al: Randomized controlled trial to determine the efficacy of early switch from vancomycin to vancomycin alternatives as a strategy to prevent nephrotoxicity in patients with multiple risk factors for adverse renal outcomes (STOP-NT). Ann Pharmacother 2017;51:185-193.

15 Sinha Ray A, et al: Vancomycin and the risk of AKI: a systematic review and meta-analysis. Clin J Am Soc Nephrol 2016;11:21322140 .

16 Molnar MZ, et al: Association of hepatitis C viral infection with incidence and progression of chronic kidney disease in a large cohort of US veterans. Hepatology 2015;61: 1495-1502.

17 Kovesdy CP, et al: Association of race with mortality and cardiovascular events in a large cohort of US veterans. Circulation 2015;132: 1538-1548.

18 George LK, et al: Association of pre-operative albuminuria with post-operative outcomes after coronary artery bypass grafting. Sci Rep 2015;5:16458.

19 Gosmanova EO, et al: Impact of non-adherence on renal and cardiovascular outcomes in US veterans. Am J Nephrol 2015;42:151157.

20 Levey AS, et al: A new equation to estimate glomerular filtration rate. Ann Intern Med 2009;150:604-612. 
21 Khwaja A: KDIGO clinical practice guidelines for acute kidney injury. Nephron Clin Pract 2012;120:c179-c184.

22 Grams ME, et al: Candidate surrogate end points for ESRD after AKI. J Am Soc Nephrol 2016;27:2851-2859.

23 Zetola N, et al: Community-acquired meticillin-resistant Staphylococcus aureus: an emerging threat. Lancet Infect Dis 2005;5: 275-286.

24 Moellering RC Jr: Vancomycin: a 50-year reassessment. Clin Infect Dis 2006;42(suppl 1):S3-S4.

25 American Thoracic SA: Infectious diseases society of, guidelines for the management of adults with hospital-acquired, ventilator-associated, and healthcare-associated pneumonia. Am J Respir Crit Care Med 2005; 171: 388-416.

26 Cano EL, et al: Incidence of nephrotoxicity and association with vancomycin use in intensive care unit patients with pneumonia: retrospective analysis of the IMPACT-HAP Database. Clin Ther 2012; 34:149-157.

27 Lodise TP, et al: Relationship between initial vancomycin concentration-time profile and nephrotoxicity among hospitalized patients. Clin Infect Dis 2009;49:507-514

28 Wunderink RG, et al: Linezolid in methicillin-resistant Staphylococcus aureus nosocomial pneumonia: a randomized, controlled study. Clin Infect Dis 2012;54:621-629.

29 van Hal SJ, Paterson DL, Lodise TP: Systematic review and meta-analysis of vancomycininduced nephrotoxicity associated with dosing schedules that maintain troughs between 15 and 20 milligrams per liter. Antimicrob Agents Chemother 2013;57:734-744.
30 Lodise TP, et al: Larger vancomycin doses (at least four grams per day) are associated with an increased incidence of nephrotoxicity. Antimicrob Agents Chemother 2008;52:13301336.

31 Bosso JA, et al: Relationship between vancomycin trough concentrations and nephrotoxicity: a prospective multicenter trial. Antimicrob Agents Chemother 2011;55:54755479.

32 Kullar R, et al: Validation of the effectiveness of a vancomycin nomogram in achieving target trough concentrations of $15-20 \mathrm{mg} / \mathrm{L}$ suggested by the vancomycin consensus guidelines. Pharmacotherapy 2011;31:441-448.

33 Pritchard L, et al: Increasing vancomycin serum trough concentrations and incidence of nephrotoxicity. Am J Med 2010;123:11431149.

34 Satoskar AA, et al: Staphylococcus infectionassociated glomerulonephritis mimicking IgA nephropathy. Clin J Am Soc Nephrol 2006;1:1179-1186.

35 Rybak MJ, et al: Vancomycin therapeutic guidelines: a summary of consensus recommendations from the infectious diseases Society of America, the American society of health-system pharmacists, and the society of infectious diseases pharmacists. Clin Infect Dis 2009;49:325-327.

36 Davis SL, et al: Adherence to the 2009 consensus guidelines for vancomycin dosing and monitoring practices: a cross-sectional survey of U.S. hospitals. Pharmacotherapy 2013;33: 1256-1263.

37 Carreno JJ, et al: Comparative incidence of nephrotoxicity by age group among adult patients receiving vancomycin. Infect Dis Ther 2013;2:201-208.
38 King DW, Smith MA: Proliferative responses observed following vancomycin treatment in renal proximal tubule epithelial cells. Toxicol In Vitro 2004;18:797-803.

39 Celik I, et al: Protective effects of different antioxidants and amrinone on vancomycin-induced nephrotoxicity. Basic Clin Pharmacol Toxicol 2005;97:325-332.

40 Toyoguchi T, et al: Nephrotoxicity of vancomycin and drug interaction study with cilastatin in rabbits. Antimicrob Agents Chemother 1997;41:1985-1990.

41 Le Moyec L, et al: Aminoglycoside and glycopeptide renal toxicity in intensive care patients studied by proton magnetic resonance spectroscopy of urine. Crit Care Med 2002;30: 1242-1245.

42 Codding CE, et al: Tubulointerstitial nephritis due to vancomycin. Am J Kidney Dis 1989; 14:512-515.

43 Wai AO, et al: Vancomycin-induced acute interstitial nephritis. Ann Pharmacother 1998; 32:1160-1164.

44 Hsu SI: Biopsy-proved acute tubulointerstitial nephritis and toxic epidermal necrolysis associated with vancomycin. Pharmacotherapy 2001;21:1233-1239.

45 Shah-Khan F, Scheetz MH, Ghossein C: Biopsy-proven acute tubular necrosis due to vancomycin toxicity. Int J Nephrol 2011;2011: 436856.

46 Belen C, et al: Biopsy-proven acute tubular necrosis associated with vancomycin in an adult patient. Ren Fail 2012:34:502505

47 Cappelletty D, Jablonski A, Jung R: Risk factors for acute kidney injury in adult patients receiving vancomycin. Clin Drug Investig 2014;34:189-193. 